\title{
Optical Lumped Element Microwave Kinetic Inductance Detectors
}

\author{
Danica Marsden ${ }^{a}$, Benjamin A. Mazin ${ }^{a}$, Bruce Bumble ${ }^{b}$, Seth Meeker ${ }^{a}$, Kieran O’Brien ${ }^{a}$, Sean \\ $\mathrm{McHugh}^{a}$, Matthew Strader ${ }^{a}$, and Eric Langman ${ }^{a}$ \\ a Department of Physics, University of California, Santa Barbara, CA, USA; \\ ${ }^{b}$ NASA Jet Propulsion Laboratory, 4800 Oak Grove Drive, Pasadena, CA, USA;
}

\begin{abstract}
Microwave Kinetic Inductance Detectors, or MKIDs, have proven to be a powerful cryogenic detector technology due to their sensitivity and the ease with which they can be multiplexed into large arrays. An MKID is an energy sensor based on a photon-variable superconducting inductance in a lithographed microresonator. It is capable of functioning as both a photon detector across the electromagnetic spectrum and a particle detector. We have recently demonstrated the world's first photon-counting, energy-resolving, ultraviolet, optical, and near infrared MKID focal plane array in the ARCONS camera at the Palomar 200" telescope. Optical Lumped Element (OLE) MKID arrays have significant advantages over semiconductor detectors such as charge coupled devices (CCDs). They can count individual photons with essentially no false counts and determine the energy (to a few percent) and arrival time (to $\approx 1 \mu \mathrm{s}$ ) of every photon, with good quantum efficiency. Initial devices were degraded by substrate events from photons passing through the Titanium Nitride (TiN) material of the resonator and being absorbed in the substrate. Recent work has eliminated this issue, with a solution found to be increasing the thickness of the TiN resonator from 20 to $60 \mathrm{~nm}$.
\end{abstract}

Keywords: MKIDs, cryogenic detectors, IFU, LTD, kinetic inductance detector, thin film, substrate event, TiN

\section{INTRODUCTION}

In the ultraviolet, optical, and near infrared wavelength range $(0.1-5 \mu \mathrm{m})$ a variety of detector technologies based on semiconductors, backed by large investment from both consumer and military customers, has resulted in detectors for astronomy with large formats, high quantum efficiency, and low readout noise. They are, however, fundamentally limited by the band gap of the semiconductor ( $1.1 \mathrm{eV}$ for silicon) and thermal noise resulting from their $\sim 100 \mathrm{~K}$ operating temperatures ${ }^{1}$. Cryogenic detectors, with operating temperatures on the order of $100 \mathrm{mK}$, allow the use of superconductors with gap parameters over 1000 times lower than typical semiconductors. This difference allows for a superconducting detector that can count single photons with no false counts, while simultaneously determining the energy (to several percent or better) and arrival time (to a microsecond) of the photon. Since the photon energy is always much greater than the gap energy, the wavelength coverage is much greater. While a CCD is limited to about $0.3-1 \mu \mathrm{m}$, the arrays described here are sensitive from $0.1 \mu \mathrm{m}$ in the UV to greater than $5 \mu \mathrm{m}$ in the mid-IR, enabling observations at infrared wavelengths vital to understanding the high redshift universe.

This approach has been pursued in the past with two technologies: Superconducting Tunnel Junctions $(\mathrm{STJs})^{2,3}$ and Transition Edge Sensors (TESs) ${ }^{4,5}$. While both of these technologies produced functional detectors, they have had difficulties with large array size due to the challenge of wiring and multiplexing large numbers of detectors. Currently the largest STJ array is 120 pixels ${ }^{6}$ and the largest optical TES array is 36 pixels $^{5}$, although recently there have been proposals for larger TES multiplexers ${ }^{7}$.

Microwave Kinetic Inductance Detectors, or $\mathrm{MKIDs}^{8}$, are an alternative cryogenic detector technology with sensitivity and ease of multiplexing initially demonstrated at millimeter wavelengths ${ }^{9,10}$. Intrinsic frequency domain multiplexing allows thousands of pixels to be read out over a single microwave cable ${ }^{11}$. The first

Further author information: (Send correspondence to D. Marsden)

D. Marsden: E-mail: danica@physics.ucsb.edu, Telephone: (805)-893-3079

High Energy, Optical, and Infrared Detectors for Astronomy V, edited by Andrew D. Holland, James W. Beletic, Proc. of SPIE Vol. 8453, 84530B - (C) 2012 SPIE · CCC code: 0277-786X/12/\$18 · doi: 10.1117/12.924904 
deployed MKID array had 1024 pixels. This number of pixels is growing, with a clear path to Megapixel arrays. Thus the ability to easily reach large formats is one of the primary advantages of MKID arrays. MKIDs can count individual photons with no false counts and determine the energy and arrival time of every photon with good quantum efficiency ${ }^{12}$. Their physical pixel size and maximum count rate are well matched with large telescopes. These capabilities enable powerful new astrophysical instruments usable from the ground and space. The first optical/nIR MKID camera, the ARray Camera for Optical to Near-IR Spectrophotometry (ARCONS), was commissioned in July, 2011 at the Palomar 200 inch telescope over 4 days ${ }^{13,14}$. During this first run, several science targets were observed, with data currently being analyzed.

In this paper we describe the first photon-counting, energy-resolving ultraviolet, optical, and near infrared MKID focal plane array, as well as progress made towards improving upon this technology. In particular, we describe the solution to the substrate event problem which prevented the first deployed array from reaching its full scientific potential. ARCONS is presently being upgraded to 2,024 of the new thicker TiN pixels, which will make it the largest optical/UV camera based on low temperature detectors by an order of magnitude.

\section{DETECTOR DESIGN}

MKIDs are lithographed superconducting resonators which have a complex surface impedance that changes when photons are absorbed into the thin metal film, breaking Cooper pairs and creating quasiparticles. Reversing the direction of the supercurrent requires extracting the kinetic energy stored in the supercurrent of a superconductor, which yields an extra inductance, an effect known as the kinetic inductance effect ${ }^{15}$. By placing a superconducting inductor in a lithographed resonator, this change can be accurately quantified, yielding the photon energy.

Each MKID pixel can be tuned to have a different resonant frequency during the fabrication process. This is accomplished by changing the total length of the inductor in a "trombone" section, resulting in a lower (higher) inductance and therefore a higher (lower) resonant frequency. A microwave probe signal tuned near the resonant frequency of the resonator is passed along the detector feedline. Photons absorbed in the inductor leave their imprint on the signal by changing the phase and amplitude of the probe signal. Since the quality factor, $Q$, of the resonators is high and their microwave transmission off resonance is nearly perfect, multiplexing is easily accomplished, with a single feedline able to read out hundreds of detectors. A comb of probe signals is sent into the device, and room temperature electronics recover the changes in amplitude and phase without significant cross talk $^{8}$, as shown in Figure 1. In this way, readout complexity is transferred to the room temperature electronics ${ }^{11}$.

The first MKID design pursued was a lumped element resonator arrangement ${ }^{16}$ shown in Figure 2 . The resonator consists of a $20 \mathrm{~nm}$ thick sub-stoichiometric titanium nitride $\left(\operatorname{TiN}_{x}\right)$ film ${ }^{17}$, with the nitrogen content tuned with $x<1$ such that the superconducting transition temperature $T_{c}$ is about $800 \mathrm{mK}$. The quasiparticle diffusion length in TiN is expected to be short (on the order of $10 \mu \mathrm{m})^{17}$. Due to the long penetration depth of these films $(\sim 1 \mu \mathrm{m})$, the surface inductance is extremely high $(90 \mathrm{pH} /$ pixel), allowing a very compact resonator fitting in a $100 \times 100 \mu \mathrm{m}$ square. Bandwidth limitations of the electronics used for the first array necessitated two feedlines for readout, each serving 512 resonators. Resonators for the first array were designed to be separated by $2 \mathrm{MHz}$ within a $4-5 \mathrm{GHz}$ band.

It was found that a double meander design for the inductor caused the electric field from the charge in each meander leg to be precisely cancelled by the adjacent $\operatorname{leg}^{18}$, minimizing crosstalk between pixels. Furthermore, the array was designed such that detectors with similar resonant frequencies were placed physically far apart. A $100 \mu \mathrm{m}$ pitch circular microlens array with an effective fill factor of $67 \%$ is used to focus the incoming light on each inductor, since photons hitting the capacitor or wiring will not be detected or will appear as photon events with an energy significantly below their true energy. The microlens produces a spot expected to have a wavelength dependent diameter of around $15 \mu \mathrm{m}$.

The responsivity of the devices depends on the current density in the metal film where the photon is absorbed. In order to achieve high energy resolution, the OLE MKID must have a uniform response to photon events. Thus in order to normalize the response, the width of each leg is tapered to give a uniform current density in the last eight legs, where the microlens spot is centered. Based on electromagnetic simulations of the current density 


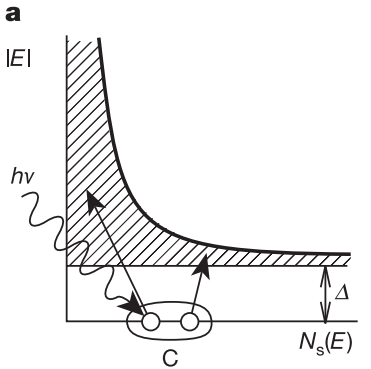

b

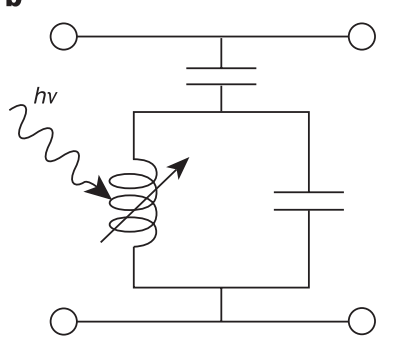

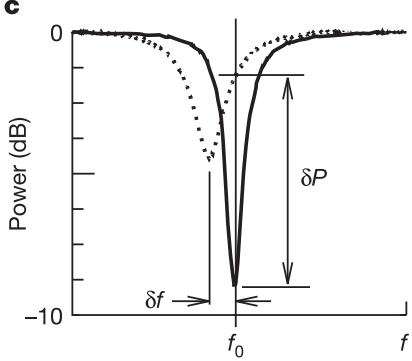

d

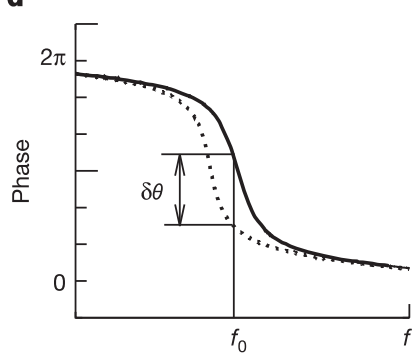

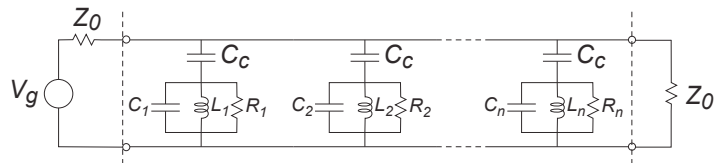

port 1

port 2

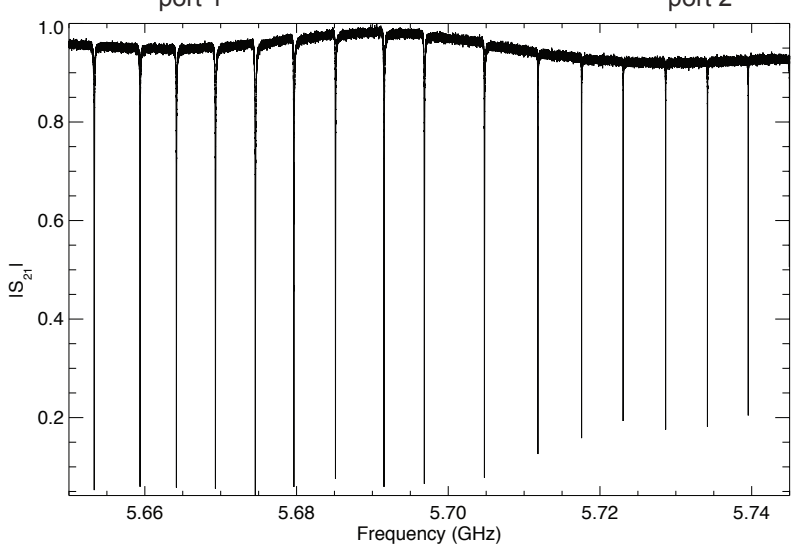

Figure 1. Left: The basic operation of an MKID. (a) Photons with energy $h \nu$ are absorbed in a superconducting film, producing a number of excitations, called quasiparticles. (b) To sensitively measure these quasiparticles, the film is placed in a high frequency planar resonant circuit. The amplitude (c) and phase (d) of a microwave excitation signal sent through the resonator. The change in the surface impedance of the film following a photon absorption event pushes the resonance to lower frequency and changes its amplitude. If the detector (resonator) is excited with a constant on-resonance microwave signal, the energy of the absorbed photon can be determined by measuring the degree of phase and amplitude shift. Right: The top panel shows the results the equivalent circuit of multiplexed MKIDs, and the bottom panel shows microwave transmission data from actual MKIDs with very accurate frequency spacing.
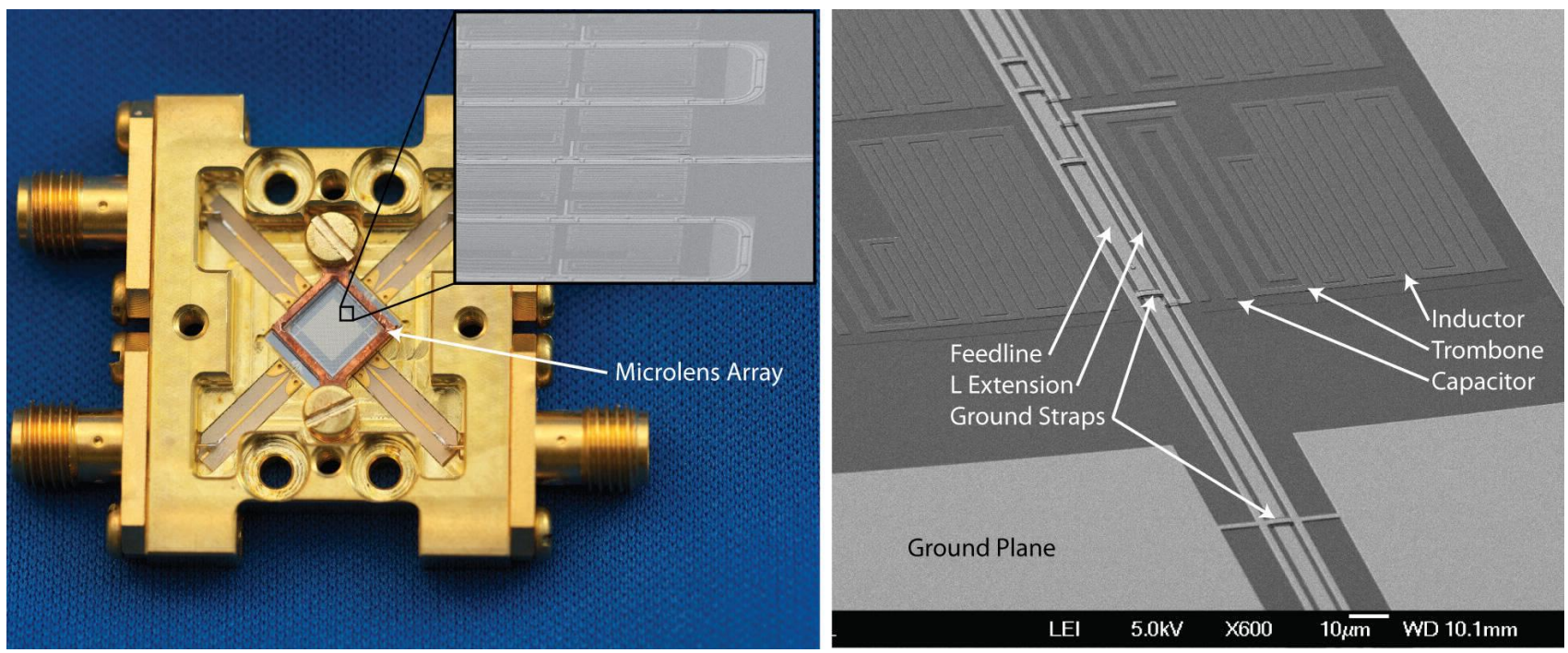

Figure 2. Left: A photograph of the 1024 pixel OLE MKID array with microlenses mounted into a microwave package. The greyscale insets are scanning electron microscope (SEM) images of the array to show the pixel design. The pixels are on a $100 \mu \mathrm{m}$ pitch, with slot widths inside the resonator of $0.5 \mu \mathrm{m}$. Right: A SEM of a OLE MKID pixel. The microwave feedline runs down the middle, with ground straps shorting the finite ground planes together. An L-shaped piece of niobium is connected to the center strip and enables strong coupling of the resonator to the feedline. The resonant frequency is adjusted by changing the length of a "trombone section". The tapering is visible as the slow increase in leg width with increasing distance from the feedline. 


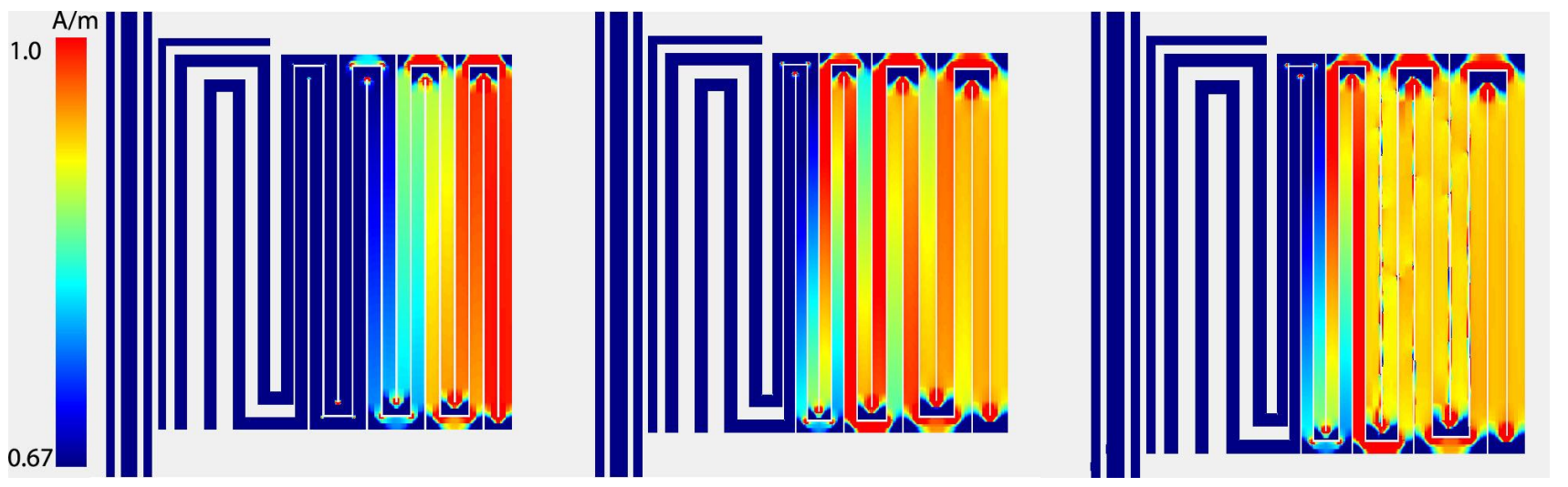

Figure 3. SONNET simulations of the resonator current density. The normalized current density is less than 0.67 in blue areas, and rises to 1.0 in the red areas. The left panel shows a resonator with uniform leg widths. The center panel show a resonators with rectangular legs with a mean width selected to give the most uniform current in each leg, and the right panel shows the final tapered resonator with fully optimized trapezoidal shaped legs.

using the SONNET software package, as shown in Figure 3, we vary the resonator leg widths from 2.5-4 $\mu \mathrm{m}$. Slots are $0.5 \mu \mathrm{m}$, primarily set by fabrication capabilities.

Early prototypes used coplanar waveguide (CPW) or coplanar slotline (CPS) feedlines to bring the probe signals to and from the resonators. These designs exhibited extreme variations in coupling quality factor $\left(Q_{c}\right.$, the strength of the resonator's coupling to the feedline) and resonant frequency, presumably due to undesired modes being excited on the feedline at discontinuities such as resonator couplers. In order to suppress these modes, a finite groundplane CPW (FCPW) feedline with regular straps that connect the ground planes together to suppress the undesired slotline mode was used instead. The feedlines are made out of Niobium since it is difficult to make $50 \Omega$ feedlines with TiN due to its high surface inductance. An extended niobium groundplane was also added to reduce crosstalk between the two feedlines. In order to achieve strong coupling (low $Q_{c}$ ) an L-shaped extension of the center strip was created.

\section{FABRICATION}

We discuss briefly the fabrication and device testing of the MKIDs, in order to provide background for Section 5, but refer the reader to Ref. 12 for further details.

The first OLE MKID science array was fabricated on a high resistivity $(10-20 \mathrm{k} \Omega \mathrm{cm}) \mathrm{Si}<100>$ wafer. The wafer was dipped in a buffered oxide etch (BOE) immediately before TiN film growth to remove the native oxide. The TiN film is deposited by reactive sputtering from a $99.99 \%$ purity Ti target in a mixture of ultra-high purity nitrogen and argon. Depositions are done at room temperature at $1 \times 10^{-7} \mathrm{~Pa}$ background pressure. The TiN film for the first deployed array was deposited at a rate of $37 \mathrm{~nm} / \mathrm{min}$ using roughly $12 \% \mathrm{~N}_{2}$ in Ar by flow at $0.266 \mathrm{~Pa}$ total pressure. Conditions were tuned to provide a sub-stoichiometric composition with slight compressive stress $(\sim 100 \mathrm{MPa}), T_{c} \sim 800 \mathrm{mK}$, and resistivity $\sim 100 \mu \Omega \mathrm{cm}$. Layers were patterned using a Canon stepping projection aligner. The TiN was reactive ion etched (RIE) with a chlorine containing gas mixture in an inductively coupled plasma system (ICP) using a photoresist mask. The wafer surface was solvent cleaned after etch step and given a mild $\mathrm{O}_{2}$ plasma clean. TiN resonators were protected by a blanket deposit of $80 \mathrm{~nm}$ RF-bias sputtered $\mathrm{SiO}_{2}$. This protection layer was then patterned and etched away from transmission line areas.

A coplanar waveguide (CPW) feedline is fabricated in three steps. The center line is sputter deposited $\mathrm{Nb}$ which is patterned by lift-off. An interlayer dielectric (ILD) consisting of $120 \mathrm{~nm}$ of $\mathrm{SiO}_{2}$ is then deposited and patterned. Finally, the surface is cleaned and patterned again for lift-off of a $160 \mathrm{~nm} \mathrm{Nb}$ film used for the ground plane metal.

The $\mathrm{SiO}_{2}$ protecting the TiN resonators is then removed. A stencil of positive resist is patterned over the CPW structure to protect the ILD. The TiN is exposed by dipping the wafer in BOE for 90 seconds. Photoresist is applied to protect the wafer for dicing into chips and not removed until the device is mounted in a sample box. 

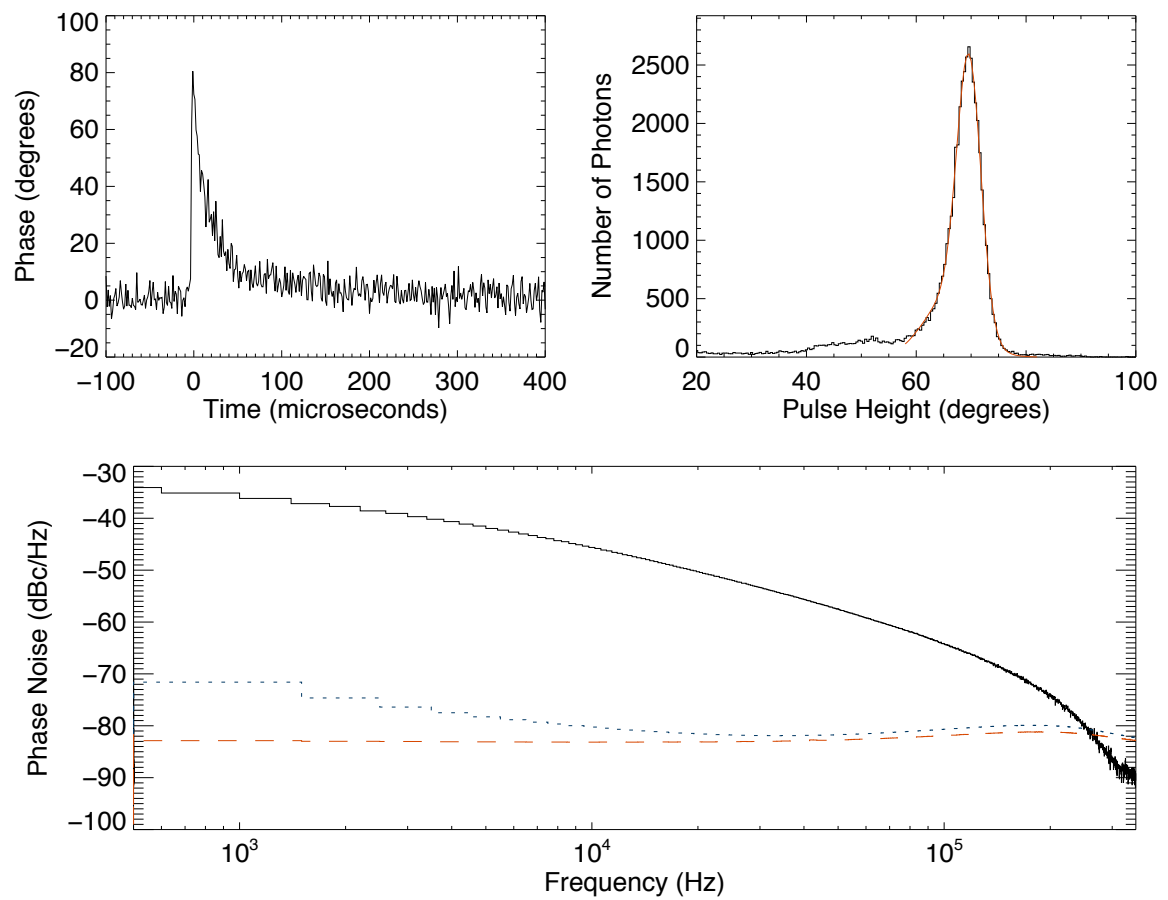

Figure 4. The top left panel shows a characteristic single photon pulse from a $254 \mathrm{~nm}$ photon striking a typical resonator with measured quality factor $Q_{m}=18,300$. The fall time of the pulse of $50 \mu$ s limits the maximum count to around 2000 counts/pixel/second. The top right panel shows a histogram of the optimally estimated pulse height based on the detection of $\sim 50,000254 \mathrm{~nm}$ photons. The solid red curve is the fit of the sum of two Gaussian to the histogram, showing an energy resolution $\mathrm{R}=16$, with a slightly broader shoulder extending to lower energies. The low energy shoulder is likely due to photons that miss the circular microlens and are absorbed in less sensitive areas of the resonator and photons that hit the substrate between the legs of the inductive meander. The bottom panel shows the Fourier transform of the average pulse template in black (arbitrary scale) as well as the measured phase (blue dotted line) and amplitude (red dashed line) noise.

Scanning electron microscope (SEM) measurements of the array showed that while there was an overall random error of $\sim 100 \mathrm{~nm}$ in the width of the slots, the widths of the legs were consistent with each other to $\sim 25 \mathrm{~nm}$. Simulations show that with these lithographic errors, non-uniform current density will not limit device energy resolution, $\mathrm{R}=\mathrm{E} / \Delta \mathrm{E}$, until $\mathrm{R}$ exceeds 80 .

\section{DEVICE TESTS}

The device was mounted in a gold-plated copper sample box and wire bonded to duroid transition boards. The sample box was inserted into a MKID testbed based on a dilution refrigerator and cooled to approximately $100 \mathrm{mK}$. A Weinreb microwave HEMT amplifier with a noise temperature of approximately $4 \mathrm{~K}$ is used to amplify the signal. The testbed allows collimated light fed from an external fiber to illuminate the array. A mercury argon light source with narrow band filters provides monochromatic light. Up to 512 resonators are probed with room temperature electronics consisting of an Anritsu signal generators, Marki IQ mixers, and a National Instruments Analog to Digitial Converter. More details on the electronics and cryogenics can be found in Refs. 19 and 11.

The response of the resonators to $254 \mathrm{~nm} \mathrm{Hg}$ line photons is shown in Figure 4. After processing with a Wiener optimal filter, an energy resolution $\mathrm{R}=16$ was measured, which matched the expected $\mathrm{R}$ based on the measured noise power spectrum and average pulse template. The theoretical energy resolution set by the creation statistics of the quasiparticles created during downconversion is $R=\frac{1}{2.355} \sqrt{\frac{\eta h \nu}{F \Delta}}$, where $\eta=0.57$ is 

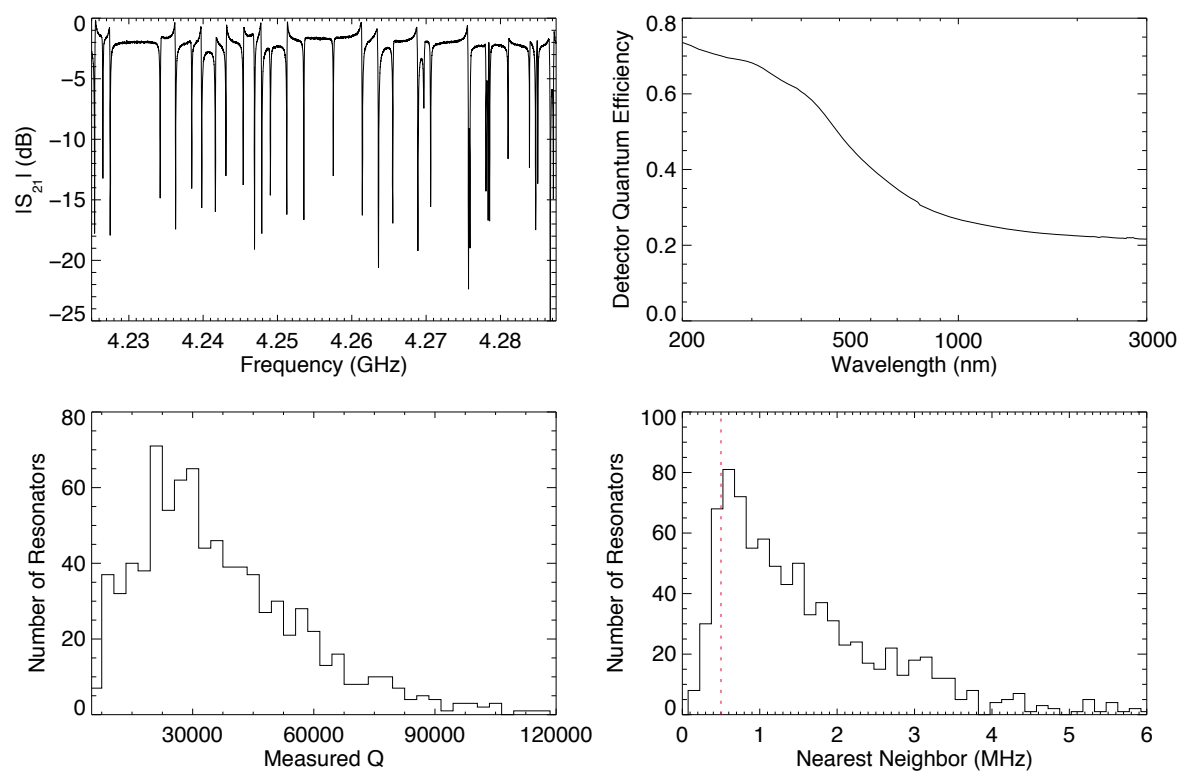

Figure 5. The top left panel shows the microwave transmission through the device over $10 \%$ of the frequency span covered by resonators. The top right panel is a measurement of the quantum efficiency of a bare $40 \mathrm{~nm}$ TiN on sapphire film. The bottom left panel is a histogram of measured quality factor for 852 out of a possible 1024 resonators. The internal quality factor of the resonators, $1 / Q_{i}=1 / Q_{m}-1 / Q_{c}$, was approximately $1 \times 10^{6}$. The bottom right panel is the frequency spacing in $\mathrm{MHz}$ between each resonator and its nearest neighbor. Most of the missing resonators are too close together in frequency $(<500 \mathrm{kHz}$, noted with a dashed line), resulting in only one resonator being included in the plot.

the efficiency of creating quasiparticles ${ }^{20}, h \nu$ is the energy of the incident photon, $\Delta=1.72 k_{B} T_{c}$ is the gap energy of the superconducting absorber, and $F \approx 0.2$ is the Fano factor ${ }^{21}$. This works out to $\mathrm{R}=150$ at $5 \mathrm{eV}$ for an operating temperature of $100 \mathrm{mK}$. An operating temperature of $15 \mathrm{mK}$ could allow a theoretical maximum energy resolution of $\mathrm{R}=400$ at $5 \mathrm{eV}$, although it is likely that other noise sources ${ }^{22}$ will become more important as future development increases the energy resolution. Therefore significant improvement can be made to increase $\mathrm{R}$ in the future.

As seen in the bottom panel of Figure 4, most of the signal from the photons comes at frequencies above $10^{4} \mathrm{~Hz}$. At these frequencies the phase and amplitude noise are nearly identical, indicating that the dominant noise source is the HEMT amplifier. The white noise level of $-83 \mathrm{dBc} / \mathrm{Hz}$ at the device readout power of -103 $\mathrm{dBm}$ is consistent with an amplifier noise temperature of 4 Kelvin assuming $3 \mathrm{~dB}$ of loss between the MKID and the amplifier. Thus reduction of the amplifier noise temperature or an increase in the maximum usable readout power should immediately improve the energy resolution.

Figure 5 shows data from a typical 32x32 pixel array. In this device, $85 \%$ of the resonators were usable. Simple number counts showed that at least $95 \%$ of the resonators were present, but variations in thickness and $\mathrm{T}_{c}$ of the TiN film caused some resonators to have similar resonant frequencies and overlap in frequency. Future improvement to the uniformity of the TiN and a more robust algorithm for placement of resonators should significantly decrease the number of overlapping resonators.

The quantum efficiency of the device was estimated by depositing a $40 \mathrm{~nm}$ TiN film on sapphire and measuring the reflection and transmission of the film as a function of wavelength, then subtracting these quantities from unity to give the fraction of photons absorbed in the superconducting film, as shown in the top right panel of Figure 5. This was done with a Varian Cary 5000 spectrometer with accessories for absolute transmission and absolute specular reflectance. This figure slightly overestimates the quantum efficiency of the final device because it does not account for losses in the microlens array, or the $\sim 10 \%$ correction due to photons below $0.5 \mu \mathrm{m}$ slipping through the slots in the inductor. The quantum efficiency is extremely good in the UV, but declines to about $30 \%$ at $1 \mu \mathrm{m}$. Further development will likely result in significant increases in quantum efficiency, with good 

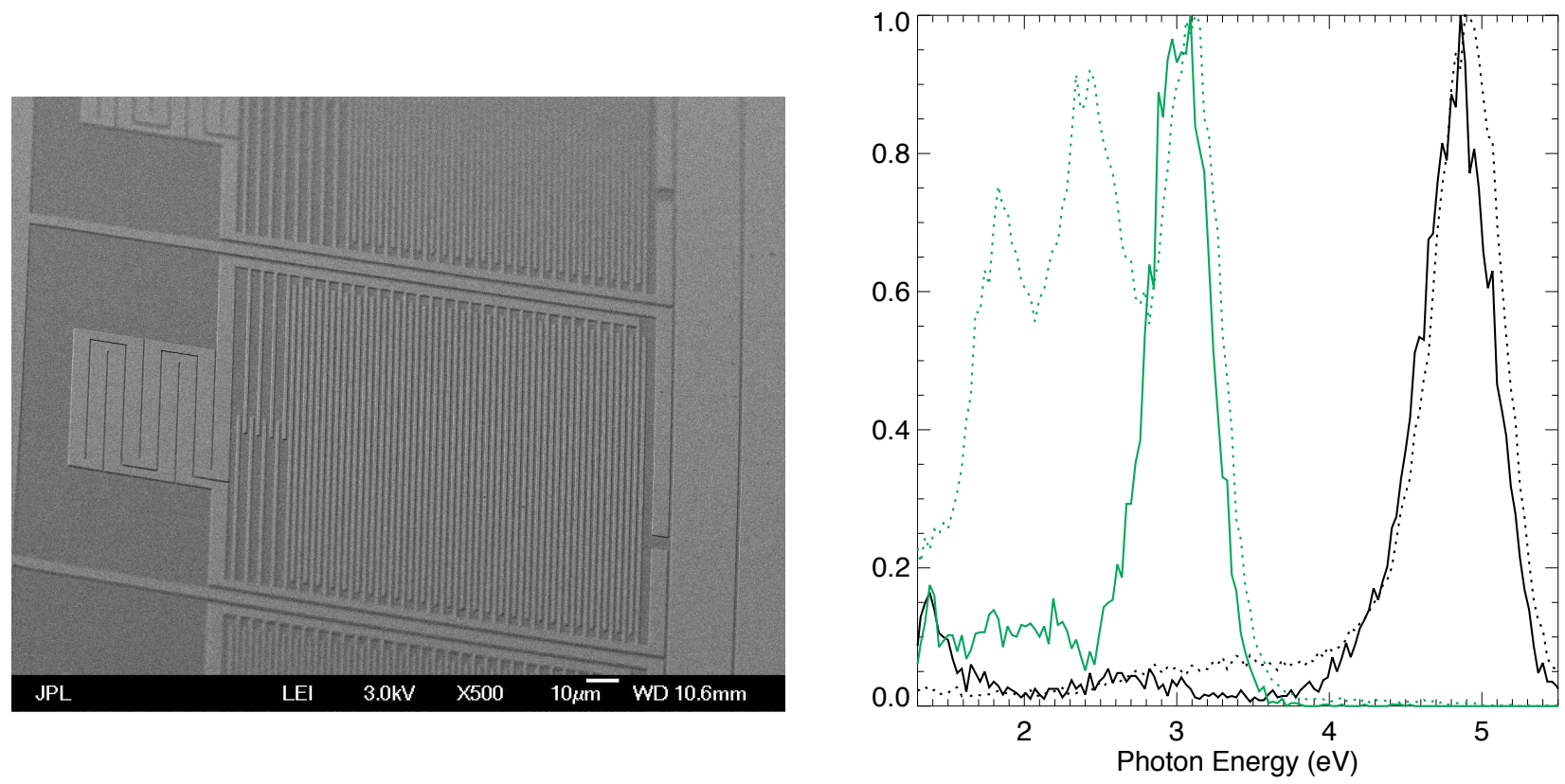

Figure 6. Left: The thicker $60 \mathrm{~nm}$ MKID design. Right: Normalized histograms of the optimally filtered pulse heights of TiN OLE MKIDs in response to stimulation with $254 \mathrm{~nm}$ (black curves) and $405 \mathrm{~nm}$ (green curves) light. The dashed lines are the results for $20 \mathrm{~nm}$ thick MKIDs, whereas the solid curves depict the response for $60 \mathrm{~nm}$ thick MKIDs. The substrate events appear as the multi-peaked, non-gaussian green dashed line for the $20 \mathrm{~nm}$ thick MKIDs when exposed to $405 \mathrm{~nm}$ light. They are clearly not present in the solid green curve, denoting the $60 \mathrm{~nm}$ thick MKIDs' response.

prospects for reaching unity. For example, we are implementing an improved lens with square lens elements that increase the fill factor to $92 \%$. As well, since light is focused to the center of the inductor with a microlens, a black absorbing spot made from a material like aluminum titanium nitride (AlTiN) or tantalum on top of the TiN in the microlens target zone in the inductor would increase photon absorption.

\section{SUBSTRATE EVENTS}

In a device with a fixed response (degrees of phase shift per eV of photon energy) the energy resolution scales linearly with photon energy, except for a region between 350 and $700 \mathrm{~nm}$ where a significant fraction of photons pass through the metal and are absorbed in the silicon substrate. These substrate events occur when a photon is absorbed close to the silicon/TiN interface, causing a significant fraction of the phonons $(>70 \%)$ created in the substrate to diffuse into the TiN and break Cooper Pairs, creating quite large signals. Ideally, the MKIDs would respond to monochromatic light in a single-peaked, Gaussian distribution. However, the substrate events cause the number counts of photons detected with a given energy to be skewed towards lower energies, and to go from single-peaked to multi-peaked, with extra peaks showing up at lower photon energies, as illustrated in Figure 6 (dotted lines).

Simple device changes, such as making the TiN film thicker or using a transparent substrate such as sapphire, should eliminate these unwanted substrate events. We tried several designs, including MKIDs with thicker TiN layers, $60 \mathrm{~nm}$ instead of $20 \mathrm{~nm}$, which is what was used for the first array. As can be seen in Figure 6 , the data for this new device, depicted by the solid curves, shows that the new design does indeed solve the substrate event problem. We are upgrading ARCONS with an array with this new design.

\section{CONCLUSION}

Improvements continue in leaps and bounds with the revolutionary MKID detectors. Better TiN film fabrication leads to increased uniformity and yield. It is reasonable to expect that the energy resolution, R, of 
the devices, currently $\approx 20$, will continue to improve towards the theoretical limit of 150 over the next several years as designs and materials evolve. The array size can be grown nearly arbitrarily by adding more resonators per feedline, likely to a maximum of around 10,000 resonators in a 10-20 GHz band, and then by adding more microwave feedlines. The primary challenges to growing the array are in the digitization bandwidth and processing power of the room temperature electronics. Furthermore, the parallel technologies of infrared-blocking filters, broadband antireflection coatings, and detector quantum efficiency continue to develop, which will increase the performance of arrays for astronomy.

OLE MKID arrays have now been proven in the lab, and the first astronomical test at the Palomar 200 inch telescope has been conducted, with data taken on a variety of astronomical objects. The ARCONS camera is currently being outfitted with a new, larger array of resonators that incorporate the improvements outlined in Sections 4 and 5. This latest design will be tested on the sky in two observing campaigns in the latter half of 2012.

This relatively new technology will bring extreme performance improvements to some of the most exciting areas of astrophysics, such as coronagraphic planet finding ${ }^{23}$, transient and time variable sources ${ }^{4}$, and high redshift galaxy evolution ${ }^{24}$, and will likely also find application in other fields such as quantum optics ${ }^{25}$ and biological imaging ${ }^{26}$.

\section{ACKNOWLEDGMENTS}

This material is based upon work supported by the National Aeronautics and Space Administration under Grant NNX09AD54G, issued through the Science Mission Directorate, Jet Propulsion Lab's Research \& Technology Development Program, and a grant from the W.M. Keck Institute for Space Studies. Part of the research was carried out at the Jet Propulsion Laboratory, California Institute of Technology, under a contract with the National Aeronautics and Space Administration. The authors would like to thank Rick LeDuc, Jonas Zmuidzinas, Sunil Golwala, David Moore, Peter Day, and Omid Noroozian for useful insights.

\section{REFERENCES}

[1] Eisaman, M. D., Fan, J., Migdall, A., and Polyakov, S. V., "Invited review article: Single-photon sources and detectors," (Jan 2011).

[2] Martin, D. D. E., Verhoeve, P., Peacock, A., Kozorezov, A. G., Wigmore, J. K., Rogalla, H., and Venn, R., "Resolution limitation due to phonon losses in superconducting tunnel junctions," Appl. Phys. Lett. 88, 3510 (Mar 2006).

[3] Hijmering, R. A., Verhoeve, P., Martin, D. D. E., Jerjen, I., Kozorezov, A. G., and Venn, R., "Direct position resolution measurement with droids at optical wavelengths," Journal of Low Temperature Physics 151, 298 (Apr 2008).

[4] Romani, R. W., Miller, A. J., Cabrera, B., Nam, S. W., and Martinis, J. M., "Phase-resolved crab studies with a cryogenic transition-edge sensor spectrophotometer," The Astrophysical Journal 563, 221 (Dec 2001). (c) 2001: The American Astronomical Society.

[5] Burney, J., Bay, T. J., Barral, J., Brink, P. L., Cabrera, B., Castle, J. P., Miller, A. J., Nam, S., Rosenberg, D., Romani, R. W., and Tomada, A., "Transition-edge sensor arrays for uv-optical-ir astrophysics," Nuclear Instruments and Methods in Physics Research Section A 559, 525 (Apr 2006).

[6] Verhoeve, P., Martin, D., Hijmering, R., Verveer, J., van Dordrecht, A., Sirbi, G., Oosterbroek, T., and Peacock, A., "S-cam 3: Optical astronomy with a stj-based imaging spectrophotometer," (Jan 2006).

[7] Niemack, M. D., Beyer, J., Cho, H. M., Doriese, W. B., Hilton, G. C., Irwin, K. D., Reintsema, C. D., Schmidt, D. R., Ullom, J. N., and Vale, L. R., "Code-division squid multiplexing," Appl. Phys. Lett. 96, 3509 (Apr 2010).

[8] Day, P., Leduc, H., Mazin, B., Vayonakis, A., and Zmuidzinas, J., "A superconducting detector suitable for use in large arrays," Nature 425, 817-821 (2003).

[9] Schlaerth, J. A., Czakon, N. G., Day, P. K., Downes, T. P., Duan, R., Gao, J., Glenn, J., Golwala, S. R., Hollister, M. I., Leduc, H. G., Mazin, B. A., Maloney, P. R., Noroozian, O., Nguyen, H. T., Sayers, J., Siegel, S., Vaillancourt, J. E., Vayonakis, A., Wilson, P. R., and Zmuidzinas, J., "Mkid multicolor array status and results from democam," Millimeter 7741, 4 (Jul 2010). (c) 2010: American Institute of Physics. 
[10] Roesch, M., Bideaud, A., Benoit, A., Cruciani, A., Désert, F. X., Doyle, S., Leclercq, S., Mattiocco, F., Schuster, K. F., Swenson, L., and Monfardini, A., "Characterization of lumped element kinetic inductance detectors for mm-wave detection," Millimeter 7741, 16 (Jul 2010). (c) 2010: American Institute of Physics.

[11] McHugh, S., Mazin, B. A., Serfass, B., Meeker, S., O’Brien, K., Duan, R., Raffanti, R., and Werthimer, D., "A readout for large arrays of Microwave Kinetic Inductance Detectors," ArXiv e-prints (Mar. 2012).

[12] Mazin, B. A., Bumble, B., Meeker, S. R., O’Brien, K., McHugh, S., and Langman, E., "A superconducting focal plane array for ultraviolet, optical, and near-infrared astrophysics," Optics Express 20, 1503 (Jan. 2012).

[13] Mazin, B. A., O’Brien, K., McHugh, S., Bumble, B., Moore, D., Golwala, S., and Zmuidzinas, J., "ARCHONS: a highly multiplexed superconducting optical to near-IR camera," in [Society of Photo-Optical Instrumentation Engineers (SPIE) Conference Series], Society of Photo-Optical Instrumentation Engineers (SPIE) Conference Series $\mathbf{7 7 3 5}$ (July 2010).

[14] O’Brien, K., Mazin, B., McHugh, S., Meeker, S., and Bumble, B., "ARCONS: a Highly Multiplexed Superconducting UV-to-Near-IR Camera," in [IAU Symposium], IAU Symposium 285, 385-388 (Apr. 2012).

[15] Mattis, D. C. and Bardeen, J., "Theory of the anomalous skin effect in normal and superconducting metals," Phys. Rev. 111, 412-417 (1958).

[16] Doyle, S., Mauskopf, P., Naylon, J., Porch, A., and Duncombe, C., "Lumped element kinetic inductance detectors," J Low Temp Phys 151, 530-536 (Jan 2008).

[17] Leduc, H. G., Bumble, B., Day, P. K., Eom, B. H., Gao, J., Golwala, S., Mazin, B. A., McHugh, S., Merrill, A., Moore, D. C., Noroozian, O., Turner, A. D., and Zmuidzinas, J., "Titanium nitride films for ultrasensitive microresonator detectors," Appl. Phys. Lett. 97, 102509 (Jan 2010).

[18] Noroozian, O., Day, P., Eom, B. H., LeDuc, H., and Zmuidzinas, J., "Microwave crosstalk in lumped element far-ir mkids," Infrared Millimeter and Terahertz Waves (IRMMW-THz), 2010 35th International Conference on , 1 (2010).

[19] Mazin, B. A., Microwave kinetic inductance detectors, PhD thesis, California Institute of Technology, California, USA (2005).

[20] Kozorezov, A. G., Wigmore, J. K., Martin, D., Verhoeve, P., and Peacock, A., "Electron energy downconversion in thin superconducting films," Phys Rev B 75, 094513 (Jan 2007).

[21] Fano, U., "Ionization yield of radiations 2: The fluctuations of the number of ions," Phys. Rev. 72(1), 26-29 (1947).

[22] Gao, J., Daal, M., Vayonakis, A., Kumar, S., Zmuidzinas, J., Sadoulet, B., Mazin, B. A., Day, P. K., and Leduc, H. G., "Experimental evidence for a surface distribution of two-level systems in superconducting lithographed microwave resonators," Appl. Phys. Lett. 92, 152505 (Jan 2008).

[23] Crepp, J. R., Pueyo, L., Brenner, D., Oppenheimer, B. R., Zimmerman, N., Hinkley, S., Parry, I., King, D., Vasisht, G., Beichman, C., Hillenbrand, L., Dekany, R., Shao, M., Burruss, R., Roberts, L. C., Bouchez, A., Roberts, J., and Soummer, R., "Speckle suppression with the project 1640 integral field spectrograph," The Astrophysical Journal 729, 132 (Mar 2011).

[24] Bouwens, R. J., Illingworth, G. D., Franx, M., and Ford, H., "z 7-10 galaxies in the hudf and goods fields: Uv luminosity functions," The Astrophysical Journal 686, 230 (Oct 2008). (c) 2008: The American Astronomical Society.

[25] Ma, L., Nam, S., Xu, H., Baek, B., Chang, T., Slattery, O., Mink, A., and Tang, X., "1310 nm differentialphase-shift qkd system using superconducting single-photon detectors," New J Phys 11, 045020 (Jan 2009).

[26] Tinoco, I. and Gonzalez, R. L., "Biological mechanisms, one molecule at a time," (Jan 2011). 